\title{
MATHEMATICAL DESCRIPTION OF THE NON-EQUILIBRIUM STATE OF SYMMETRIC PARTICLE SYSTEMS
}

\author{
Halyna M. Hubal \\ Computer Science and Information Technologies Faculty \\ Lutsk National Technical University \\ 75 Lvivs'ka Str., Lutsk, 43018 - UKRAINE
}

\begin{abstract}
One-dimensional symmetric hard-sphere system is considered in this paper. Non-equilibrium state of this system is described by the configuration distribution function that is the solution of the Cauchy problem to the equation of the diffusion type (the speed distribution is the Maxwell one and is unchanged).
\end{abstract}

AMS Subject Classification: 35Q82

Key Words: BBGKY hierarchy of equations, Maxwell speed distribution, symmetric particle system, non-equilibrium state

\section{Introduction}

The mathematical basis of the description of the evolution of dynamical systems of particles (hard spheres) is the BBGKY hierarchy of equations (see [1], [2], [3], [4]). However, in some cases, simpler approaches can be applied. In particular, these approaches are applied to one-dimensional system of hard spheres.

The main feature of such a system is that there are no changes of velocities of colliding particles, there is exchange of velocities. Thus, there is no change in the speed distribution function. This function is specified by an initial distribution which can be arbitrary. For example, as the initial distribution, we choose the

Received: June 30, 2019

(C) 2019 Academic Publications 
Maxwell speed distribution:

$$
\varphi(v)=\frac{1}{\sqrt{2 \pi} \sigma} e^{-\frac{v^{2}}{2 \sigma^{2}}},
$$

where $\sigma=\sqrt{\frac{k T}{m}}$ is a root-mean-square speed.

\section{Formulation of the problem}

Consider one-dimensional symmetric system of $N$ identical particles (hard spheres) with the mass $m=1$ and the diameter $2 d>0$ moving along the straight line with a length of $L$. Each particle is characterized by the coordinate of the centre $q_{i}$ of the sphere and by its momentum $p_{i}$, i.e. $\left(q_{i}, p_{i}\right)=x_{i}$.

By the Gibbs hypothesis, the equilibrium distribution has the form

$$
f\left(x_{1}, \ldots, x_{N}\right)=\Xi^{-1} e^{-\beta H_{N}^{L}}
$$

where $\beta$ is inversely proportional to the temperature $T$, the statistical sum

$$
\Xi=\int_{\left(L \times R^{1}\right)^{N}} d x_{1} \ldots d x_{N} e^{-\beta H_{N}^{L}},
$$

the Hamiltonian

$$
H_{N}^{L}=\sum_{i=1}^{N} \frac{p_{i}^{2}}{2}+\sum_{i=1}^{N-1} \Phi\left(\left|q_{i}-q_{i+1}\right|\right)+\sum_{i=1}^{N} u^{L}\left(q_{i}\right),
$$

the pair hard-core interaction potential

$$
\Phi(|q|)= \begin{cases}+\infty, & \left|q_{i}-q_{i+1}\right| \leq 2 d \\ 0, & \left|q_{i}-q_{i+1}\right|>2 d\end{cases}
$$

the set of forbidden configurations

$$
\begin{gathered}
W_{N}=\left\{\left(q_{1}, \ldots, q_{N}\right) \in L:\left|q_{i}-q_{i+1}\right| \leq 2 d\right. \text { at least for a single pair } \\
\left.\left(q_{i}, q_{i+1}\right), i=\overline{1, N-1}\right\}
\end{gathered}
$$

the set of permissible configurations

$$
L \backslash W_{N}=\left\{\left(q_{1}, \ldots, q_{N}\right) \in L:\left|q_{i}-q_{i+1}\right|>2 d, i=\overline{1, N-1}\right\},
$$


the holding potential

$$
u^{L}(q)= \begin{cases}+\infty, & q<d, q>L-d \\ 0, & d<q<L-d .\end{cases}
$$

For this system, we assume that:

- initial velocities $v_{i}$ and coordinates $q_{i}$ of particles are independent random variables;

- speed distributions are not degenerate;

- the mathematical expectation $\bar{v}_{i}=0$ and the function $\varphi\left(v_{i}\right)$ is even. Nearest neighbours interaction is typical for this system. However, collisions lead to the exchange of velocities which, in fact, eliminates mixing of velocities. Thus, the speed distribution function is unchanged.

Let the particles be located in the interval $\left[-\frac{L}{2}, \frac{L}{2}\right]$ at the initial moment of time. Assuming that $L \rightarrow \infty$, we neglect the influence of the boundaries on the particle motion. In this case, the distribution function of the system state at arbitrary moment of time $t$ can unambiguously be expressed in terms of the distribution function at $t=0$ :

$$
D\left(q_{1}, v_{1} ; q_{2}, v_{2} ; \ldots ; t\right)=D_{0}\left(q_{1}-v_{1} t, v_{1} ; q_{2}-v_{2} t, v_{2} ; \ldots\right),
$$

where $\left(q_{i}-v_{i} t, v_{i}\right)$ is the system state at $t=0,\left(q_{i}, v_{i}\right)$ is the system state at arbitrary moment of time $t$. This relation underlines the fact that onedimensional symmetric system is the system of non-interacting particles.

Thus, the behaviour of the system can be described by considering the motion of each particle independently from each other.

We assume that particle sizes tend to zero because

- we can consider particle sizes in the initial distribution function $D_{0}$ (the presence of forbidden configurations),

- the coordinates of centers can be redetermined to consider particle sizes.

The concentration can be considered a small fixed value and the average distance $\frac{1}{n}$ between particles can be considered significantly larger than particle sizes, i.e. $\frac{1}{n} \gg 2 d$. 


\section{The kinetic equation}

Let $F(q, v, t)$ is the distribution function of the probability that at the moment of time $t$ there is a particle moving with the velocity $v$ near the point $q$. Since particles are identical this probability is equal to the probability that at the moment of time $t=0$ there was a particle with the velocity $v$ near the point $q=v t$, i.e.:

$$
F(q, v, t) d q d v=F(q-v t, v, 0) d q d v .
$$

Let $F(q, v, t)=f(q, t) \varphi(v)$. Then

$$
f(q, t) \varphi(v) d v=f_{0}(q-v t) \varphi_{0}(v) d v .
$$

Integrating the last equality with respect to $v$, we obtain

$$
f(q, t)=\int_{-\infty}^{\infty} f_{0}(q-v t) \varphi_{0}(v) d v
$$

i.e. the distribution at the moment of time $t$ is determined by the initial distribution.

Let the speed distribution function have Maxwell's form at the initial moment of time, i.e.

$$
\varphi_{0}(v)=\frac{1}{\sqrt{2 \pi} \sigma} e^{-\frac{v^{2}}{2 \sigma^{2}}},
$$

and particles be located in some region at the initial moment of time, i.e. we assume that

$$
f_{0}(q)=\frac{1}{\sqrt{2 \pi} \delta} e^{-\frac{q^{2}}{2 \delta^{2}}},
$$

where $\delta$ is the size of the region of localization. Then

$$
\begin{gathered}
f(q, t)=\int_{-\infty}^{\infty} f_{0}(q-v t) \varphi_{0}(v) d v \\
=\frac{1}{\sqrt{2 \pi} \delta} \frac{1}{\sqrt{2 \pi} \sigma} \int_{-\infty}^{\infty} e^{-\frac{(q-v t)^{2}}{2 \delta^{2}}} e^{-\frac{v^{2}}{2 \sigma^{2}}} d v \\
=\frac{1}{2 \pi \delta \sigma} \int_{-\infty}^{\infty} e^{-\frac{v^{2}-\frac{2 \sigma^{2} q t}{\sigma^{2} t^{2}+\delta^{2}} v+\frac{\sigma^{2} q^{2}}{2 \delta^{2} \sigma^{2}}}{\sigma^{2}+\delta^{2}}}\left(\sigma^{2} t^{2}+\delta^{2}\right) d v
\end{gathered}
$$




$$
=\frac{1}{\sqrt{2 \pi} \sqrt{\sigma^{2} t^{2}+\delta^{2}}} e^{-\frac{q^{2}}{2\left(\sigma^{2} t^{2}+\delta^{2}\right)}}
$$

is the Gauss distribution with increasing variance. Putting $\delta=0$, we get the Green function

$$
G\left(q, q_{0}, t\right)=\frac{1}{\sqrt{2 \pi} \sigma t} e^{-\frac{\left(q-q_{0}\right)^{2}}{2 \sigma^{2} t^{2}}}
$$

which is the fundamental solution of the equation of the diffusion type:

$$
\frac{\partial f}{\partial t}=\sigma^{2} t \frac{\partial^{2} f}{\partial q^{2}}
$$

where $\sigma=\sqrt{\frac{k T}{m}}$ is a root-mean-square speed.

Statistics of the description of non-equilibrium state of the system is given by the following theorem.

Theorem 1. Non-equilibrium state of one-dimensional symmetric hardsphere system is described by the configuration distribution function that is the solution of the Cauchy problem to the equation of the diffusion type (1) (the speed distribution is the Maxwell one and is unchanged).

Proof. Let us find the solution of the Cauchy problem

$$
\left\{\begin{array}{l}
\frac{\partial f}{\partial t}=\sigma^{2} t \frac{\partial^{2} f}{\partial q^{2}}, \\
f(0, q)=f_{0}(q) .
\end{array}\right.
$$

The solution can be represented in the form of factorized product:

$$
f(t, q)=\Phi(t) Q(q) \neq 0
$$

then

$$
\begin{gathered}
\Phi^{\prime}(t) Q(q)=\sigma^{2} t \Phi(t) Q^{\prime \prime}(q), \\
\frac{\Phi^{\prime}(t)}{\sigma^{2} t \Phi(t)}=\frac{Q^{\prime \prime}(q)}{Q(q)}=-\lambda^{2},
\end{gathered}
$$

where $\lambda=$ const. Then

$$
\left\{\begin{array}{l}
\Phi^{\prime}(t)+\lambda^{2} \sigma^{2} t \Phi(t)=0, \\
Q^{\prime \prime}(q)+\lambda^{2} Q(q)=0,
\end{array}\right.
$$


whence we obtain the differential equation

$$
\frac{d \Phi(t)}{\Phi(t)}=-\lambda^{2} \sigma^{2} t d t
$$

Integrating this equation, we obtain

$$
\Phi_{\lambda}(t)=C_{1}(\lambda) e^{-\frac{(\lambda \sigma t)^{2}}{2}} .
$$

Similarly, we get

$$
Q_{\lambda}(q)=C_{2}(\lambda) \cos \lambda q+C_{3}(\lambda) \sin \lambda q .
$$

The particular solution has the form

$$
f_{\lambda}(t, q)=e^{-\frac{(\lambda \sigma t)^{2}}{2}}(A(\lambda) \cos \lambda q+B(\lambda) \sin \lambda q) .
$$

Then the general solution has the form

$$
f(t, q)=\int_{-\infty}^{\infty} e^{-\frac{(\lambda \sigma t)^{2}}{2}}(A(\lambda) \cos \lambda q+B(\lambda) \sin \lambda q) d \lambda
$$

whence for $t=0$, we obtain

$$
f_{0}(q)=\int_{-\infty}^{\infty}(A(\lambda) \cos \lambda q+B(\lambda) \sin \lambda q) d \lambda
$$

The Fourier integral for $f_{0}(q)$ can be written in the form

$$
\begin{gathered}
f_{0}(q)=\frac{1}{2 \pi} \int_{-\infty}^{\infty} d \lambda \int_{-\infty}^{\infty} f_{0}\left(q_{0}\right) \cos \lambda\left(q-q_{0}\right) d q_{0} \\
=\frac{1}{2 \pi} \int_{-\infty}^{\infty}\left(\cos \lambda q \int_{-\infty}^{\infty} f_{0}\left(q_{0}\right) \cos \lambda q_{0} d q_{0}+\sin \lambda q \int_{-\infty}^{\infty} f_{0}\left(q_{0}\right) \sin \lambda q_{0} d q_{0}\right) d \lambda
\end{gathered}
$$

then

$$
A(\lambda)=\frac{1}{2 \pi} \int_{-\infty}^{\infty} f_{0}\left(q_{0}\right) \cos \lambda q_{0} d q_{0}, \quad B(\lambda)=\frac{1}{2 \pi} \int_{-\infty}^{\infty} f_{0}\left(q_{0}\right) \sin \lambda q_{0} d q_{0}
$$


Therefore,

$$
\begin{gathered}
f(t, q)=\frac{1}{2 \pi} \int_{-\infty}^{\infty} d \lambda \int_{-\infty}^{\infty} e^{-\frac{(\lambda \sigma t)^{2}}{2}} f_{0}\left(q_{0}\right) \cos \lambda\left(q-q_{0}\right) d q_{0} \\
=\frac{1}{\pi} \int_{-\infty}^{\infty} f_{0}\left(q_{0}\right) d q_{0} \int_{0}^{\infty} e^{-\frac{(\lambda \sigma t)^{2}}{2}} \cos \lambda\left(q-q_{0}\right) d \lambda \\
=\frac{1}{\sigma t \sqrt{2 \pi}} \int_{-\infty}^{\infty} f_{0}\left(q_{0}\right) e^{-\frac{\left(q-q_{0}\right)^{2}}{2 \sigma^{2} t^{2}}} d q_{0}
\end{gathered}
$$

since $\int_{0}^{\infty} e^{-\lambda^{2} a^{2}} \cos \beta \lambda d \lambda=\frac{\sqrt{\pi}}{2 a} e^{-\frac{\beta^{2}}{4 a^{2}}}$. In particular, the fundamental solution is the Green function $G\left(q, q_{0}, t\right)$ considered above. The theorem is proved.

Remark 1. If $f_{0}\left(q_{0}\right)=\frac{1}{L}$, then

$$
f(t, q)=\frac{1}{\sigma t \sqrt{2 \pi}} \lim _{L \rightarrow \infty} \frac{N}{L} \int_{-L / 2}^{L / 2} e^{-\frac{\left(q-q_{0}\right)^{2}}{2 \sigma^{2} t^{2}}} d q_{0}=\frac{N}{L}=n
$$

i.e. $f(t, q)$ has the sense of concentration.

In general, if $f_{0}\left(q_{0}\right) \rightarrow$ const, then $f(t, q) \rightarrow$ const, i.e. $f(t, q)$ is the uniform distribution which is equilibrium one. An estimation of relaxation time has the form $t \approx L \sqrt{\frac{m}{k T}}$.

\section{Conclusions}

We proved that non-equilibrium state of one-dimensional symmetric hard-sphere system is described by the configuration distribution function that is the solution of the Cauchy problem to the equation of the diffusion type (1) (the speed distribution is the Maxwell one and is unchanged). 


\section{References}

[1] N.N. Bogolyubov, Problems of a Dynamical Theory in Statistical Physics, Gosudarstv. Izdat. Tehn. Teor. Lit., Russian Federation (1946) [in Russian].

[2] C. Cercignani, V.I. Gerasimenko, D.Ya. Petrina, ManyParticle Dynamics and Kinetic Equations, Kluwer Acad.

Publ., Netherlands (1997);

doi: http://dx.doi.org/10.1007/978-94-011-5558-8.

[3] G.N. Gubal', On the existence of weak local in time solutions in the form of a cumulant expansion for a chain of Bogolyubov's equations of a onedimensional symmetric particle system, J. of Mathematical Sciences, 199 (2014), 654-666; doi: http://dx.doi.org/10.1007/s10958-014-1892-1.

[4] M.A. Stashenko, G.N. Gubal', Existence theorems for the initial value problem for the Bogolyubov chain of equations in the space of sequences of bounded functions, Siberian Math. J., 47, No 1 (2006), 152-168; doi: http://doi.org/10.1007/s11202-006-0015-8. 